\title{
Prescribing Pattern of Medications in Geriatric Patients in a South Indian Tertiary Care Teaching Hospital
}

\author{
Pavani Golla', Ranga Rao Bheemathati ${ }^{2}$, Sai Pawan Adepu Ramesh', Namratha Edara', Ramesh \\ Adepu ${ }^{1, *}$
}

${ }^{1}$ Department of Pharmacy Practice, Vikas College of Pharmaceutical Sciences, Suryapet, Telangana, INDIA. ${ }^{2}$ KIMS Hospital, Sreepuram, Narketpalli, Telangana, INDIA.

\begin{abstract}
Background: Globally, the population of elderly is gradually rising. Co-morbidities and polypharmacy are the prime reasons for the inappropriate use of medications. Objective: A prospective, observational study was conducted in a South Indian tertiary care teaching hospital to assess the medication prescribing pattern in elderly patients and also to evaluate the inappropriateness of medications using Beer's criteria. Materials and Methods: Institutional ethics committee has approved the study. Written informed consent was taken from all the enrolled elderly patients meeting the inclusion criteria. Necessary demographic, clinical, laboratory and therapeutic information was collected and recorded in a suitably designed data collection form and the same was evaluated for prescribing pattern and inappropriateness using Beer's criteria. Results: A total of 104 patients aged above 60 years were enrolled into the study. Among them 64 patients (61.53\%) were males and 40 (38.46\%) were females. Among these patients $61(58.65 \%)$ patients were in the age group of $60-65$ years, $25(24.03 \%)$ were in $66-70$ years, $12(11.53 \%)$ were in $71-75$ years, $6(5.76 \%)$ were $>75$ years. Common cause for hospital admission was hypertension and diabetes. As per Beer's criteria, $91.3 \%$ prescriptions given to the study patients were found inappropriate. Antibiotics and Pantoprazole were the most commonly prescribed drugs. The study findings suggest that use of poly pharmacy is high and majority medications prescribed were found inappropriate as per the Beer's criteria. Conclusion: Presence of clinical pharmacist would optimize the drug therapy and minimize the drug related problems and negative therapeutic outcomes in elderly patients.
\end{abstract}

Key words: Geriatrics, Beer's criteria, Inappropriate medication, Polypharmacy, Irrational prescribing.

\section{INTRODUCTION}

Geriatrics is the branch of medicine that deals with the elderly people health care. ${ }^{1}$ Rational prescribing is defined as use of least number of medicines to obtain the best possible therapeutic outcomes in the shortest period and at an affordable cost. Prescribing Pattern Monitoring Studies (PPMS) focus on prescribing, dispensing and distribution of medicines. The main aim of PPMS is to facilitate Rational use of Medicines (RUM). ${ }^{2}$ Inappropriate drug prescribing is a global problem and irrational use of drugs is becoming major concern of presentday medical practice as its consequences leading to therapy ineffectiveness, drugrelated problems and increased medical expenditure. $^{3}$
Five important criteria in rational drug use are accurate diagnosis, proper prescribing, correct dispensing, suitable packing, patients' adherence to their medication. Study of prescribing pattern seeks to monitor, evaluate and if necessary suggest modifications in prescribing so as to make medical care rational and cost effective. ${ }^{4}$ Following are the factors that affect the choice of medicines in elderly such as poly pharmacy, altered drug response, inappropriate prescribing, non-adherence to the prescribed medicines. Irrational drug use, precipitates negative therapeutic outcomes and also drains family savings. ${ }^{5}$

Elderly people suffer commonly from these diseases such as osteoarthritis, cardiovascular
DOI: 10.5530/ijopp.13.1.11

Address for correspondence: Dr.Ramesh Adepu, Principal cum Director Vikas College of Pharmaceutical Sciences, Suryapet, Telangana, INDIA.

Phone no: +91-9945520215 Email Id: adepu63@gmail.com

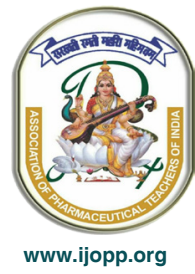


diseases, cerebrovascular diseases, osteoporosis, infectious diseases, nutritional related issues, alcohol liver diseases, gastritis or constipation, psychiatry related disorders and cancer. ${ }^{6}$ Elderly people constitute a heterogeneous group due to age related changes in pharmacokinetic and pharmacodynamic profiles of drugs. ${ }^{7}$ Changes that occur with aging are decreased GI absorption, skin absorption, shrinkage of liver, decrease in hepatic blood flow, also the body fat increases in proportion to water and muscle, fat soluble drugs undergo slower elimination, impaired renal function and reduced GFR. ${ }^{8}$ Other characteristics such as co-morbidities and polypharmacy, drug related problems, medication non-adherence also show influence on medication prescribing. Thus it is always a challenge and complex process in prescribing medicines for geriatric patients.

Polypharmacy is the major cause for inappropriate prescribing and therefore increases the risk of drug interaction related adverse drug events, disability, hospitalization, inefficient utilization of resources and even death. ${ }^{8,9}$ For the safe and appropriate use of medicines in an elderly, Mark H. Beers, a geriatrician created the "Beers criteria" in 1991 and a consensus panel of experts modified it in 1997, 2003, 2012 and 2015 using Delphi method. ${ }^{10}$ Beers criteria aims to provide the guidelines to the users about inappropriate drug names and their potential side effects.

The Beers criteria consist of medications to be avoided in the elderly irrespective of patient's diagnosis and those that should be avoided due to drug-disease, drugsyndrome interactions and those drugs that should be used with caution in older. According to Beers Criteria, drugs which are prescribed inappropriately are classified into one of the following categories:

Category A: Drugs that generally should be avoided in older adults.

Category B: Drugs that exceed maximum recommended daily dose.

Category C: Drugs to be avoided in combination with specific co-morbidity.

The present study is conducted to analyze the prescribing pattern in elderly and assess inappropriate medicines using Beers criteria among elderly patients in the study site.

\section{MATERIALS AND METHODS}

The study was a prospective observational study conducted at in-patient ward of geriatric department in a 1050 bed tertiary care teaching hospital and the study was approved by institutional ethics committee. Patients willing to participate were enrolled after taking their written informed consent. Patients were observed daily from day one of their admission till the day of their discharge and the demographic and treatment details were recorded in a separate data collection form. Patients aged sixty years and above were included in to the study. After obtaining the requisite permission, prescriptions were copied on random basis to minimize the bias. The information such as case ID, sex, age, weight, date of consultation, diagnosis and all the drugs prescribed with their doses, dosage forms, route of administration, total duration of drugs was recorded and analyzed. Collected data was entered into a suitable statistical program and the recorded data was analyzed. The statistical analysis was done by using excel software package.

\section{RESULTS}

About 104 elderly patients meeting the inclusion criteria were enrolled in to the study. Among the enrolled patients, 64 were males and 40 were female patients. About 61 $(58.65 \%)$ patients were in the age group of $60-65$ years, $25(24.03 \%)$ patients were in the age group of $66-70$ years, $12(11.53 \%)$ patients were in the age group of $71-75$ and $6(5.76 \%)$ patients were in the age group of $>76$ years among them $3(2.88 \%)$ were males and $3(2.88 \%)$ were females. Results are presented in Figure 1.

Clinical conditions of the study patients were analyzed. The results reveal that, hypertension (18 cases) is the common clinical condition followed by 15 hypertension with diabetes mellitus cases, 4 cases of Hypertension with chronic kidney disease, one case each of Alcoholic liver disease, Breast carcinoma, Hypertension with CVA, Hypertension with Diabetes mellitus and CVA and Parkinsonism and Hypertension with Diabetes mellitus with chronic kidney disease. The results are presented in Figure 2.

\section{Assessment of prescription inappropriate using Beers criteria}

Medicines received by the study patients was analyzed for inappropriateness. The results suggest that $91 \%$ of the study patients received potentially inappropriate medications. Medicines falls under the category of Potentially Inappropriate Medications (PIMs) in the prescriptions were studied. The study findings suggest that, proton pump inhibitors $74(71.15 \%)$ were the most commonly prescribed medicines followed by Diuretics $25(24.03 \%)$, oral hypoglycemics 23 (22.11\%), Calcium channel blockers 10 (9.61\%), HMG Co A reductase inhibitors $9(8.65 \%)$, NSAIDs 8 (7.69\%), $\mathrm{H}_{2}$ receptor 
antagonists $6(5.76 \%)$, anti-cholinergic drugs 3 (2.88\%), anti-Parkinson medications $2(1.92 \%)$, followed by anti infective, alpha 1 blockers, alpha 1 agonists. Results are presented in Figure 3.

Number of drugs per prescription was assessed. The results reveal that, $15(14 \%)$ prescriptions contain 1-5 drugs, 52 (50\%) prescriptions contain 6-10 drugs, $33(32 \%)$ prescriptions possess $11-15$ drugs and 4 prescriptions contain 16-20 drugs. The findings are presented in Figure 4.

Severity level of medications belonging to potentially inappropriate was assessed. Anti-infective agents such as Nitrofurantoin is categorized as Mild. NSAIDs such as Aspirin, HMG Co A Reductase Inhibitors such as Atorvastatin, Diuretics such as furosemide, Spiranolactone, $\mathrm{H}_{2}$ Receptor antagonist such as

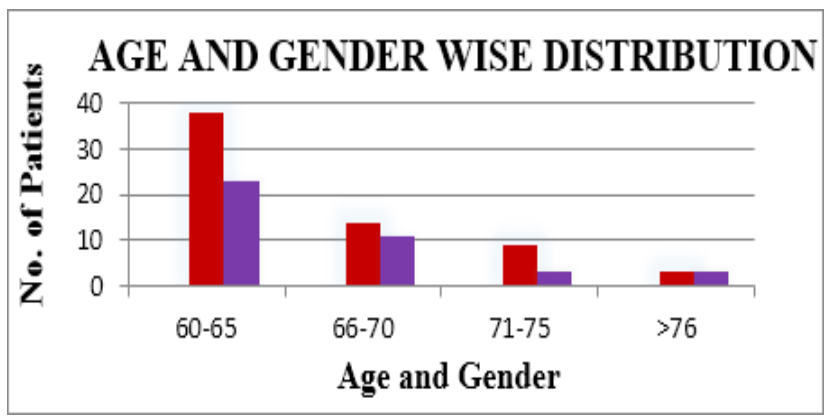

Figure 1: Age and gender wise distribution.

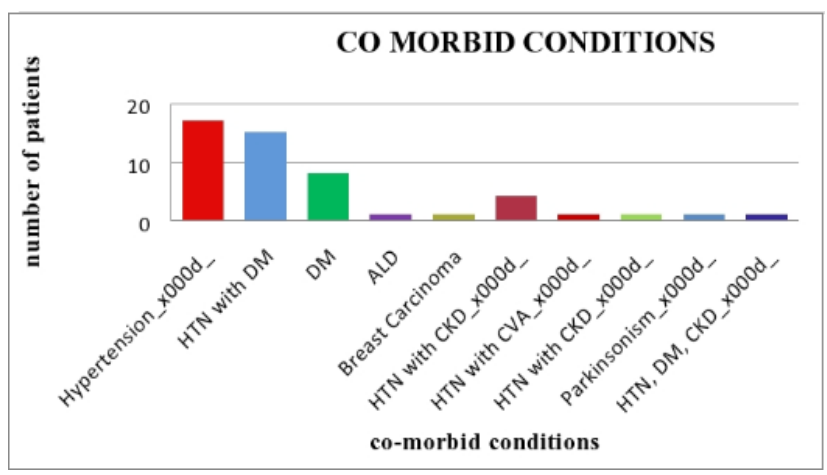

Figure 2: Clinical Conditions present in the study patients.

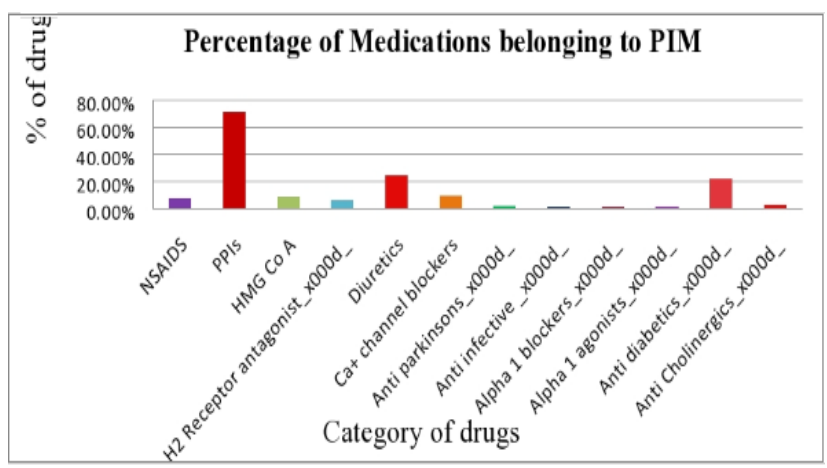

Figure 3: Percentage of medicines belonging to PIMs.

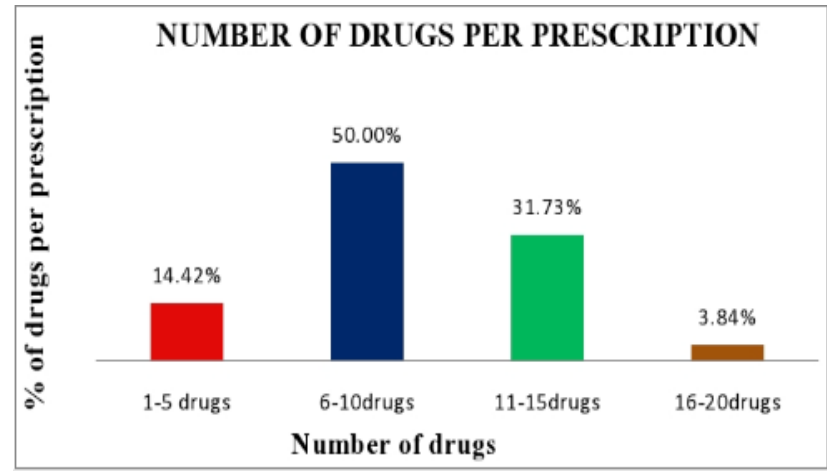

Figure 4: Number of drugs per prescriptions.

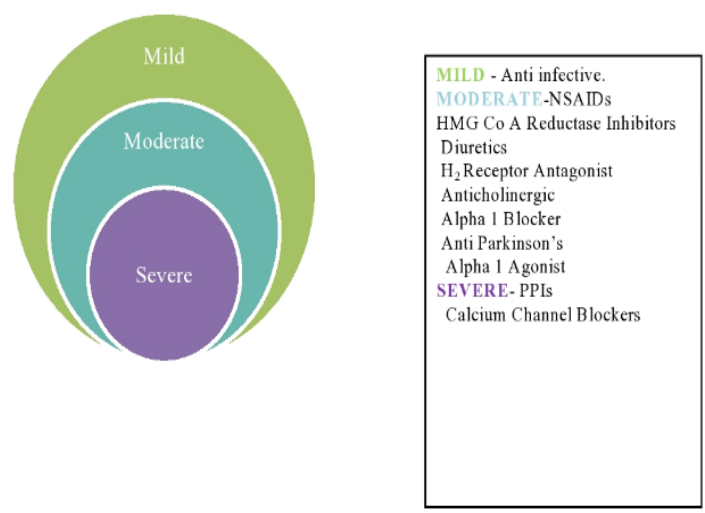

Figure 5: Assessment of Severity level of Potentially inappropriate medications.

Ranitidine, Anticholinergic such as Chlorpheniramine, alpha 1 blocker such as Prazosin, anti-Parkinson's such as Trihexiphenidyl, Alpha 1 agonist such as phenylephrine are classified as Moderate. Proton pump inhibitors such as pantoprazole, Calcium channel blocker such as Nifedipine are categorized as severe as per the 2015 updated Beer's Criteria. The findings are presented in Figure 5.

\section{DISCUSSION}

Ageing of population is rapidly increasing throughout the world. According to $66^{\text {th }}$ World Health Assembly organized by WHO in May 2013, an increase in the population was observed from $11 \%$ in 2000 to $22 \%$ in 2050 as per the proportion of people aged over 60 years. ${ }^{6}$ Many studies have revealed that patients are being exposed for unnecessary therapy for long term, leading to development of adverse drug reactions and taxing the patients in terms of physical, economical and psychological well-being. Studies also have demonstrated the prevalence of potentially inappropriate medicines use in elderly. The findings suggest that, highest prevalence of PIM was observed in France (66\%) and least was observed is in India with $17.9 \%$. It was also observed that, prevalence increases with settings, co-morbidities and polypharmacy. ${ }^{7}$

Indian Journal of Pharmacy Practice, Vol 13, Issue 1, Jan-Mar, 2020 
In our setting, the most frequently prescribed medicines are Proton pump inhibitors $(71.15 \%)$ with pantoprazole ranking as the highest among them. Pantoprazole was probably being prescribed for prophylaxis for NSAID induced gastritis. Normally the proton pump inhibitors are used for long term to manage GORD conditions in elderly. ${ }^{11}$ Though PPI are considered safe but they possess risk especially in elderly. Studies have revealed that their use has been associated with increased risks of adverse events, such as bone fractures, kidney disease, microscopic colitis and hypomagnesaemia ${ }^{12}$ Use of PPIs has also been suggested to cause changes in the composition of the intestinal microbiota, increasing the risk of Clostridium difficile infection and chronic liver disease. Although PPIs have demonstrated to minimize NSAID-related adverse effects in the stomach, recent evidence suggests that PPIs might cause changes in the composition of the small intestinal microbiota, augmenting unwanted adverse effects of NSAIDs in the small intestines. Furthermore, discontinuation of PPI treatment has been linked to acid hyper secretion and the development of dyspeptic symptoms in healthy volunteers. ${ }^{13}$ Proton pump inhibitors also interact with drugs like ketoconazole; diazepam, ciprofloxacin and clopidogrel which are commonly prescribed in the elderly to reduce their therapeutic efficacy. ${ }^{14}$ These findings pose questions about the rationality of PPIs use in elderly.

In our study, the second most frequently prescribed medications are diuretics (24.03\%). Diuretics are prone to cause electrolyte imbalance. Especially in elderly, use of diuretics are to treat hypertension or congestive heart failure or ankle edema. Many studies have observed the misuse of diuretics as clinical diagnosis was not appropriately established. Due to diuretics induced adverse effects and drug interactions patients' clinical conditions are worsened. ${ }^{15,16}$ It was also observed that, in elderly patients more than $50 \%$ of diuretics induced adverse effects are preventable in nature. ${ }^{17}$ This observation projects the need of therapeutic review by clinical pharmacist to minimize the potentially inappropriate medications.

The third most prescribed medications are oral hypoglycemic agents and insulin $(22.11 \%)$. The incidence of DM in the geriatric population is very high and are at high risk of hypoglycemia with intensive glycemic control. Such initiatives have severe consequences such as falls and traumatic fractures, exacerbation of co morbidities with adverse cardiovascular events, impaired cognition and function. Few observational studies have demonstrated mortality even in patients with near normal $A_{1 C}(<6 \%)$ goal. Medicines induced dizziness, weakness and confusion and adrenergic manifestations such as sweats, tremor are needed to be monitored. ${ }^{18}$

Calcium channel blockers $(9.61 \%)$ were the fourth most prescribed drugs in the elderly to manage hypertension. The incidence of hypertension in geriatric population is very high and is a significant determinant of cardiovascular risk in this group. HMG-CoA reductase inhibitors $(8.65 \%)$ are the fifth most prescribed drugs followed by Anticholinergics (2.88\%), antiparkinsons $(1.92 \%)$, alpha 1 agonist, alpha 1 blocker, NSAIDs. In an observational study to assess the potentially inappropriate antihypertensive medications, it was observed more than $70 \%$ of patients receive inappropriate antihypertensive medications leading to cardiovascular damage and death. ${ }^{19}$ The above findings suggest the need for clinical accuracy checking of the medications. Presence of Clinical pharmacist at bedside will help in minimizing the drug related problems and inappropriateness of medications.

Large numbers of drugs were prescribed by brand names because the prescribers are more conversant with the brand names than generic names of drug products although they are economical. Pressure from the medical representatives also contribute immensely for brand prescribing. Irrational drug use adds economic burden to the patients who have to buy the branded products at a higher cost than a generic with the same bioequivalence.

In the present study $91.34 \%$ of prescriptions met Beer's criteria for inappropriate prescribing in the elderly. These findings are significantly different from that found in a study from India. ${ }^{7}$ In which it was observed that the use of inappropriate medicine is $11.3 \%, 43.5 \%$ respectively. The reasons for inappropriate prescribing may be partly due to the relatively weak evidence-based guidelines for appropriate prescribing in elderly patients and existence of particulars justifying exceptions to the rules in individual patients.

Inappropriate use of medication in patients, 65 years and above has been linked to many adverse drug interactions; poor physical functioning and excess health care cost. Justifiable interventions will add value in appropriate drug selection by physicians.

Inappropriate high-risk drugs should be avoided in the elderly as they hamper the desired therapeutic outcomes in the patients. Use of five or more medications is considered as polypharmacy, which was observed in our study. Highest percentage of drugs per prescription was found to be $50.00 \%$. Similar data was also found in some other similar studies by Taskeen et al..$^{3}$ Prakash Goudanavar et al. ${ }^{19}$ The rise in polypharmacy may be due to more literate elderly patients or their care takers. 


\section{CONCLUSION}

Prescribing for the elderly was found to be suboptimal and there was occurrence of inappropriate prescribing. This calls for caution on the part of prescribers and pharmacists alike and also the need for awareness of tools that can be used by practitioners for detecting drug therapy problems. In order to improve drug therapy in geriatrics, it is very important for the prescribers to make themselves aware about Beer's criteria and that it should be strictly followed for better health outcomes in the elderly. More studies are required on the pattern of inappropriate prescribing over a long period of time and on intervention programs to reduce potentially adverse health outcomes in elderly patients. A key role can be played by the pharmacist to manage drug therapy in collaboration with prescribers which will be very effective in preventing the adverse drug reactions that may be resulting from inappropriate medications in geriatric patients.

\section{ACKNOWLEDGEMENT}

Authors expresses sincere thanks to the managements of KIMS Hospital, Vikas College of Pharmaceutical Sciences, Dr. Shruthi Mohanthi, Principal, Kamineni Institute of Medical Sciences, Narketpally, Dr. Martin Luther, Medical Superintendent, Dr. Shouri Reddy, General Manager, KIMS Hospital for support and encouragement in successful completion of the research work.

\section{CONFLICT OF INTEREST}

Authors declare that they have no conflict of interest.

\section{ABBREVIATIONS}

PPMS: Prescribing Pattern Monitoring Studies; RUM: Rational Use of Medicines; PIM: Potential Inappropriate Medicines.

\section{SUMMARY}

- Globally, elderly population is gradually rising and suffer from age related co-morbidities.

- To manage various clinical conditions, many medications are prescribed.

- In elderly, many medicines become inappropriate due to changed pharmacokinetics and pharmacodynamics.

- Beer's criteria is globally used to assess the inappropriateness of the medications in elderly patients. As per Beer's criteria drugs are categorised in to A, B and C.

- During the study period, a total of 104 patients aged above 60 years were enrolled into the study. Among them 64 patients $(61.53 \%)$ were males and $40(38.46 \%)$ were females. Among these patients 61(58.65\%) were in the age group of $60-65$ years, $25(24.03 \%)$ were in 66-70 years, $12(11.53 \%)$ were in $71-75$ years, $6(5.76 \%)$ were
$>75$ years. Common cause for hospital admission was hypertension and diabetes.

- As per Beer's criteria, $91.3 \%$ prescriptions given to the study patients were found inappropriate.

- Antibiotics and Pantoprazole were the most commonly prescribed drugs.

- The study findings suggest that use of polypharmacy is high and majority medications prescribed were found inappropriate as per the Beer's criteria.

- Study concludes that presence of clinical pharmacist would optimize the drug therapy and minimize the drug related problems and negative therapeutic outcomes in elderly patients.

\section{REFERENCES}

1. https://www.google.co.in/ search?hl=en and ie=ISO-1 and $q=$ geriatrics+definition

2. Vikas S. A Systematic review of prescription pattern monitoring studies and their effectiveness in promoting rational use of medicines. Perspect Clin Res. 2015;6(2):86-90.

3. Mushina T, Anitha N, Syed RA, Rao B, Abdul BK. A Study of rational drug prescribing pattern in geriatric patients in Hyderabad. Journal of Drug Delivery and Therapeutics. 2012;2(5):109-13.

4. Parthasarathi G, Nyfort-Hansen K, Milap CN. A Text book of Clinical Pharmacy Practice, Essential Concepts and Skills, $2^{\text {nd }}$ Edition, Chapter 16-Clinical Pharmacy for Geriatric patients. 2004;265-89.

5. Ross-Degnan D, Laing RO, Quick JD, et al. A strategy for promoting improved pharmaceutical use: The International Network for Rational Use of Drugs. Soc Sci and Med. 1992;35(11):1329-41.

6. Romana A, Jayanthi CR. Polypharmacy leading to ADRs in elderly in a tertiary care hospital. International Journal of Pharma and Biosciences. 2012;3(3):21824.

7. Harugeri, Joseph J, Parthasarathi G, Ramesh M, Guido S. Potentially inappropriate medication use in elderly patients: A study of prevalence and predictors in two teaching hospitals. J Postgraduate Med. 2010;56(3):186-91.

8. Swathi B. Pattern of medication use among elderly patients attending medicine department in a tertiary care hospital in India. Asian Journal of Pharmaceutical and Clinical Research. 2016;9(6):266-9.

9. Lourdu JA, Venkata NKP, Udayalakshmi P, et al. Drug utilization patterns of geriatric patients admitted in the medicine department of a tertiary care hospital. International Journal of Pharmacy and Life Sciences. 2013;4(11):3087-92.

10. American Geriatrics Society 2015 Beers Criteria Update Expert Panel. American Geriatrics Society 2015 Updated Beers Criteria for potentially inappropriate medication use in older adults. J Am Geriatr Soc. 2015;63(11):2227-46. Doi: 10.1111/jgs.13702. PMID26446832.

11. Hani AS, Khalid YS, Abdul VM, et al. Prescribing pattern of drugs in the geriatric patients in Jazan Province, KS. Pharm Pharmacol Int J. 2015;2(1):30-4.

12. Óskar ÖH, Anton $P$, Einar SB, et al. Proton pump inhibitors-A nationwide drug utilization study. Ther Adv Gastroenterol. 2018;11:1-11.

13. Marlicz W, Łoniewski I, Grimes DS, et al. Nonsteroidal anti-inflammatory drugs, proton pump inhibitors and gastrointestinal injury: Contrasting interactions in the stomach and small intestine. Mayo Clin Proc. 2014;89(12):1699-709.

14. Ogawa R, Echizen H. Drug-drug interaction profiles of proton pump inhibitors. Clin Pharmacokinet. 2010;49(8):509-33.

15. James K, John C. Inappropriate use of loop diuretics in elderly patients. Age and Aging. 2000;29(6):489-93.

16. Richard WP, Gordana G, Steven KSW, John RM. Reducing the risk of adverse drug events in older Adults. Am Fam Physician. 2013;87(5):331-6.

17. Louise K, Leah S, Leonid B. Treating the elderlydiabetic patient: Special considerations. Diabetes Metab Syndr Obes. 2014;7:391-400.

18. Marquez PHP, Torres $\mathrm{OH}$, San JA, et al. Potentially inappropriate Antihypertensive Prescriptions to Elderly Patients: Results of a Prospective Observational Study. Drugs Aging. 2017;34(6):453-6.

19. Prakash G. A Prospective study on medication prescribing pattern for geriatric patients. Asian Journal of Biomedical and Pharmaceutical Sciences. 2016;6(56):23-7. 